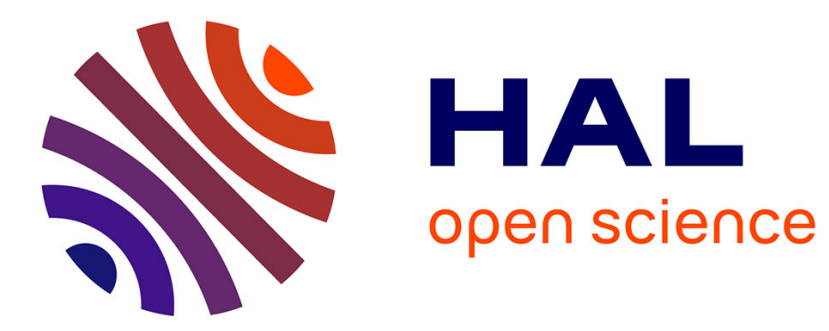

\title{
Role of Fingerprint Mechanics and non-Coulombic Friction in Ultrasonic Devices
}

Eric Vezzoli, Ddzidek Brigyda, Thomas Sednaoui, Frédéric Giraud, Adams Michael, Betty Lemaire-Semail

\section{- To cite this version:}

Eric Vezzoli, Ddzidek Brigyda, Thomas Sednaoui, Frédéric Giraud, Adams Michael, et al.. Role of Fingerprint Mechanics and non-Coulombic Friction in Ultrasonic Devices. IEEE - World Haptics Conference 2015, Jun 2015, Chicago, United States. 10.1109/WHC.2015.7177689 hal-01253582

\section{HAL Id: hal-01253582 \\ https://inria.hal.science/hal-01253582}

Submitted on 11 Jan 2016

HAL is a multi-disciplinary open access archive for the deposit and dissemination of scientific research documents, whether they are published or not. The documents may come from teaching and research institutions in France or abroad, or from public or private research centers.
L'archive ouverte pluridisciplinaire HAL, est destinée au dépôt et à la diffusion de documents scientifiques de niveau recherche, publiés ou non, émanant des établissements d'enseignement et de recherche français ou étrangers, des laboratoires publics ou privés. 


\title{
Role of Fingerprint Mechanics and non-Coulombic Friction in Ultrasonic Devices *
}

\author{
Eric Vezzoli, Brygida Dzidek, Thomas Sednaoui, Frédéric Giraud, Member, IEEE, \\ Michael Adams, and Betty Lemaire-Semail, Member, IEEE
}

\begin{abstract}
Ultrasonic vibration of a plate can be used to modulate the friction of a finger pad sliding on a surface. This modulation can modify the user perception of the touched object and induce the perception of textured materials. In the current paper, an elastic model of finger print ridges is developed. A friction reduction phenomenon based on non- Coulombic friction is evaluated based on this model. Then, a comparison with experimental data is carried out to assess the validity of the proposed model and analysis.
\end{abstract}

\section{INTRODUCTION}

Current touch based user interfaces lack natural or at least credible haptic feedback, for example, when interacting with a virtual keyboard, which was one of the main issues during the initial commercialization of smartphones. Haptic interaction is still limited to the vibration of the whole device, but is ineffective in recreating the sensation expected from an image on the screen. Few approaches have shown a realistic potential for coupling with the flat capacitive position sensing displays that are employed in consumer devices. One involves the use of a lateral vibrating surface to simulate the vibrotaction signal of the fingertip induced by sliding over a real texture [1]. Another is based on the modulation of the friction between a sliding finger and the active surface. The possibility of tracking the position of a finger led to the generation of a spatio-temporal relationship that is useful for simulating texture [2]. Stimulating the whole fingertip area in the same manner led to the classification of this approach as global stimulation. Electrovibration and ultrasonic vibration are the two leading technologies for coupling with capacitive touch screens. The former is based on the polarization of the finger pad with a high voltage supplied plate covered with an insulator [3]-[5], the resulting attractive force results in an increased friction while interacting with the display. The latter involves out-of-plane ultrasonic vibration to attenuate the friction between the contacting finger pad and the display. The generation of a squeeze film effect between a vibrating plate and the finger pad was the first mechanistic interpretation [6], [7]. It relied on a simplified model of the interaction between two smooth rigid surfaces because of the enormous complexity of a real finger pad-display interaction e.g. the large range of Knudsen numbers involved and the viscoelastic deformation of a finger pad. There is evidence that squeeze flow is an important contributory factor [8], modeled by Biet et al.[9], but an analysis of the mechanical and tribological interactions between a finger pad and an ultrasonic vibrating plate has never been performed. This is essential because the coefficients of friction associated with such devices can be $>0.1$, which is greater than that which would be expected by an effective acoustic levitation mechanism $(<0.01)$; intermittent finger pad contacts have been confirmed recently [10]. The aim of the current work is to introduce a first order mechanical model of the finger pad that is applicable to the ultrasonic domain. It is clear that the model is not complete, especially it neglects inertia and viscosity of the fingerprints. Nevertheless it can help to establish key principles to be further extended. Firstly, the mechanical interplay between the proposed model with a lateral vibrating ultrasonic plate is evaluated and a psychophysical assessment is proposed to assess the relevance of the approach. Secondly, the interaction with a normal ultrasonic vibrating plate is analyzed and the reduction of the friction with a smooth surface is estimated.

\section{Finger PAD TRIBOLOGY}

Provided that a finger pad is sufficiently moist, particularly due to occlusion of moisture secreted from the sweat pores in the finger print ridges when in contact with a smooth impermeable surface such as glass, the friction may be described by stochastic molecular theories that have been developed for elastomers [11]. Friction arises from the intermittent extension and rupture of molecular junctions at the sliding interface, which involves dangling macromolecular chains; such bonds are continually pinning and depinning due to thermal activation. A characteristic of elastomers is that they exhibit aging such that the adhesion to a rigid substrate increases with the dwell time. This arises because molecular rearrangements occur at the interface and the associated relaxation times are coupled to those for pinning. Thus disruption of the pinning process will result in a reduction of the friction. Frictional aging for finger pads is an extreme example that occurs over tens of seconds due to the plasticization of the asperities on the finger pad ridges by the secreted moisture, which results in an increase in the contact area [12]. It is reasonable to suppose that this is accompanied by aging associated with the formation of molecular bonds.

\footnotetext{
* This work was funded by the European Union under the FP7 programs FP7-PEOPLE-317100 PROTOTOUCH.

E. Vezzoli, F. Giraud and B. Lemaire-Semail are with L2EP-IRCICA Laboratory, University of Lille 1, Lille, France, 59650 (phone: +33 362531632; email: eric.vezzoli@ed.univ-lille1.fr, frederic.giraud@polytech-lille.fr, betty.semail@polytech-lille.fr).

B. Dzidek and M. Adams are at the School of Chemical Engineering, University of Birmingham, Edgbasto, B152TT, United Kingdom (e-mail: b.m.dzidek@bham.ac.uk,m.j.adams@bham.ac.uk).

T. Sednaoui is with L2EP-IRCICA and STMicroelectronics, Crolles F38920, France (e-mail: thomas.sednaoui@st.com).
} 


\section{LATERAL UltRASONIC ViBRATIONS}

\section{A. Introduction}

In this section, the influence of lateral vibration on a finger pad under a small tangential load, which is less than that required for slip, is considered. It is proposed that the friction is reduced by micro-slip due to the disruption of the molecular bond network. Such slip requires the elastic retractive force induced in the finger pad by the periodic displacement to be greater than the frictional force. It is probable that, at low vibrational frequencies, this displacement will be completely accommodated by the gross deformation of the finger pad. However, the finger pad is viscoelastic so that the stiffness increases with frequency [13]; at sufficiently high frequencies it will be assumed that (a) the response is entirely elastic and (b) the finger pad is sufficiently rigid that the deformation is localized to the finger print ridges.

\section{B. Bed of springs approximation}

The elastic response of a finger pad will be modelled as a bed of springs as shown schematically in Fig. 1. Different models have been proposed based on a combination of springs or springs and damper [14], but no one focused on the behavior of the fingertip in the ultrasonic domain. The springs may be treated as finger print ridges for current purposes.

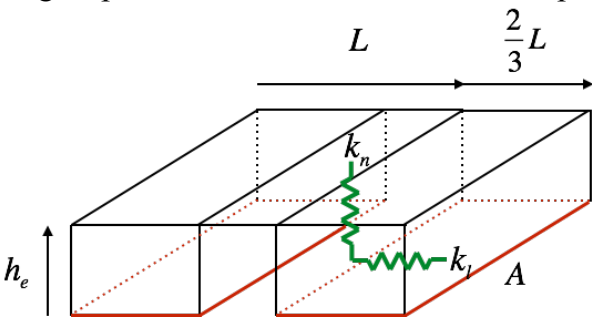

Figure 1: Bed of springs model.

The normal stiffness, $k_{n}$, is defined by:

$$
k_{n}=\frac{E_{e} A}{h_{e}}
$$

where $E_{e}$ is the Young modulus of the stratum corneum, $A$ is the contact area between the plate and the ridges and $h_{e}$ is the undeformed height of the ridges. The tangential stiffness, $k_{l}$, is defined by:

$$
k_{l}=\frac{G_{e} A}{h_{e}} \quad \text { and } \quad G_{e}=\frac{E_{e}}{2(1+v)}
$$

where $G_{e}$ is the shear modulus and $v$ is the Poisson modulus of the ridges. The dependence of $A$ on the normal force, $F_{n}$, is assumed to be:

$$
A=k\left(F_{n}\right)^{\frac{2}{3}}
$$

where $k$ is equal to $138.1 \mathrm{~mm}^{2} / \mathrm{N}^{\frac{2}{3}}$ (this value is the result of a best fit to the experimental data reported in [15]).

TABLE I

PARAMETERs VALUE

\begin{tabular}{ccc}
\hline & Parameter & Value \\
\hline i & $E_{e}$ & $1 \mathrm{MPa}$ \\
ii & $v$ & 0.4 \\
iii & $h_{e}$ & $150 \mu \mathrm{m}$ \\
iv & $\rho$ & $900 \mathrm{Kg} / \mathrm{m}^{3}$ \\
\hline
\end{tabular}


The other parameters considered in the analysis are given in table 1 . Thus, the value of $k_{l}$ could be calculated from (2) using that of $A$ obtained from (3).

\section{Modelling slip}

The criteria for stick and slip are given by the following expressions:

$$
\begin{array}{ll}
k_{l} \Delta \mathrm{x}_{\text {max }} \leq \mu_{s} F_{n} & \text { No slip } \\
k_{l} \Delta \mathrm{x}_{\text {max }}>\mu_{d} F_{n} & \text { Slip }
\end{array}
$$

where $\Delta \mathrm{x}_{\max }$ is the maximum displacement of the springs induced by the lateral vibrating plate, $\mu_{s}$ is the static coefficient of friction and $F_{n}$ is the applied normal force across the bed of springs. If the slip condition is satisfied, there is relative displacement when $k_{l} \Delta x>\mu_{s} F_{n}$ until the relative velocity between the finger pad and the plate is zero, $V=0$, Fig. 2 . In the represented case, $\mu_{s}$ is 1 , the dynamic coefficient of friction $\mu_{d}$ is 0.7 , and the applied normal force is $0.5 \mathrm{~N}$. The calculation ignores the offset tangential preload force, which could not be measured in the current work. If it is greater than the force induced by the periodic displacement, then no slip will occur in the direction of the offset force and slip will be enhanced in the other direction, so that the model will correspond to a lower bound solution. In Fig 2, the case for an ultrasonic harmonic vibration of $20 \mathrm{kHz}$ of $5 \mu \mathrm{m}$ for an applied force of $0.5 \mathrm{~N}$ is represented.

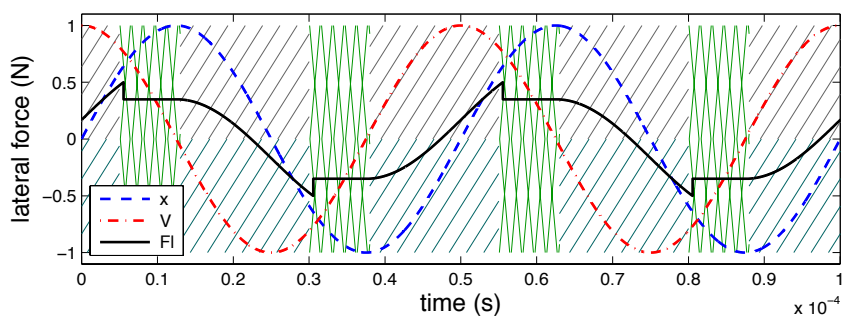

Figure 2: Steady state lateral sliding where $x$ is the normalized position of the plate, $V$ is the normalized speed of the plate and $F_{l}$ is the lateral force experienced by the finger. In the gray shaded area, the relative motion between the ridges and the plate is zero. In the green crossed area the finger is sliding on the plate.

A glide threshold is defined as the minimum amplitude of vibration necessary to induce a transition from stick to slip as represented in Fig. 2:

$$
\Delta \mathrm{x}_{t}=\frac{\mu_{s} F_{n}}{k_{l}}
$$

where $\Delta \mathrm{x}_{t}$ is the critical threshold displacement. The contact area between the finger pad and the plate is dependent on the applied normal force (3) and this dependence influences the value of $k_{l}$ (2). It is possible to compute the dependence of the threshold displacement on the applied normal force by solving (4) for small time steps to calculate the associated lateral displacements, $\delta x$, with the limit defined by (5) as shown in Fig. 3a.

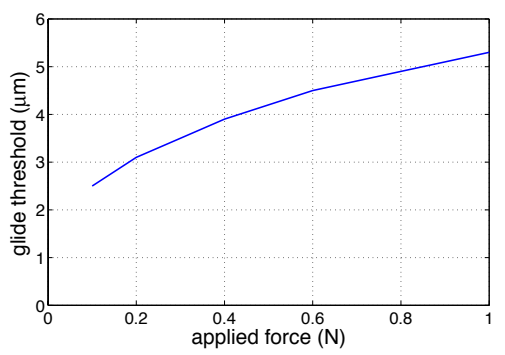

(a)

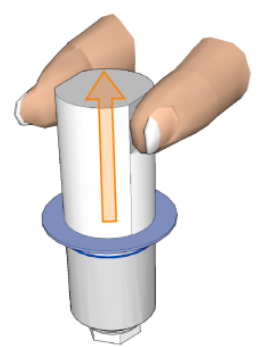

(b)

Figure 3: Glide threshold as a function of the applied force (a) and experimental setup for psychophysical evaluation (b).

\section{Psychophysical validation}

1) Experimental setup

In order to validate the model, it is necessary to evaluate the induced tactile response of a lateral vibrating plate. A Langevin actuator (FBL28302SSF-FC, Fuji Ceramics Corporation, Japan) was employed with a closed-loop vibration amplitude stabilization system to ensure the stability of the vibration amplitude during the experiment. The employed actuator has a resonance frequency of $27.83 \mathrm{kHz}$ at ambient temperature. The control of the piezoelectric actuator was implemented with piezoceramic sensor glued on the body. A DSP (EZDSPF2812, Texas Instrument, USA) was programmed do obtain the desired 
behavior of the device. The actuator has two parallel surfaces at the top of the device $\left(2 \times 1.4 \mathrm{~cm}^{2}\right)$ on both sides for making contact with the finger pad, Fig $3 \mathrm{~b}$.

2) Methododolgy and results

Four participants (male, age between 26 and 41) were asked to describe their tactile perception while holding the actuator as in Fig. $3 \mathrm{~b}$ for vibrational amplitudes of 0,2 and $4 \mu \mathrm{m}$ after imposing a small tangential force on the vibrator that was insufficient to induce gross slip. All participants gave their informed consent to perform the experiment. They were equipped with a headset to cancel the influence of environmental noise and they were asked to close their eyes during the experiments. The frequency of the stimulation was the resonance of the actuator at $27.83 \mathrm{kHz}$. The amplitudes of vibration were changed randomly and the participants reported their corresponding tactile evaluation. None of the participants could identify a difference between the 2 $\mu \mathrm{m}$ amplitude and the static condition. However, all reported a sensation of slip for an amplitude of $4 \mu \mathrm{m}$ with comments such as "it felt more slippy." This amplitude corresponds to the glide threshold for an applied normal force of about $0.4 \mathrm{~N}$, which is typical of the values used in touch [15].

\section{NORMAL VIBRATIONS}

\section{A. Introduction}

In this section, the interaction of the proposed model with a normal vibrating plate is proposed, and the friction reduction arising from the interaction with a smooth surface is estimated. It has been shown that an ultrasonic vibrating plate in the normal direction can induce intermittent contact with a finger pad for vibrational amplitudes of a few micrometres [10]. This attach/detach phenomenon could lead to a friction reduction during the sliding motion of the finger due to the influence on the interfacial molecular bonding interactions in addition to the development of a gaseous squeeze film. Here, the loading/unloading cycle of a finger pad again will be represented by a bed of equivalent elastic springs to investigate the mechanism in more detail. A key factor is that the detachment will lead to rupture of the interfacial bonds and thus finite time is required for repinning. It is assumed that the viscoelastic retardation time of the finger pad is infinitely long during the unloading cycle of an out-of-plane imposed vibration compared to the reciprocal frequency of the vibration. The finger print ridges are much stiffer than the finger pad and also it is assumed that they will behave elastically during unloading.

\section{B. Bed of springs model}

Following a compression cycle of a continuous out-of-plane vibration, the springs will unload and detachment will require the vibration amplitude to be greater than the unloaded length of the spring. The critical amplitude to induce detachment, $W_{c}$, is given by the following expression for the current model:

$$
W_{c}=\Delta \mathrm{z}_{n}=\frac{F_{n}}{k_{n}}
$$

where $\Delta \mathrm{z}_{n}$ is the compression induced by the normal load and $k_{n}$ was calculated from (1). The dependence of the necessary vibrational amplitude to induce detachment of the fingertip as a function of the applied force in the range relevant to touch is reported in Fig 4. The analysis is valid for frequencies greater than $20 \mathrm{kHz}$.

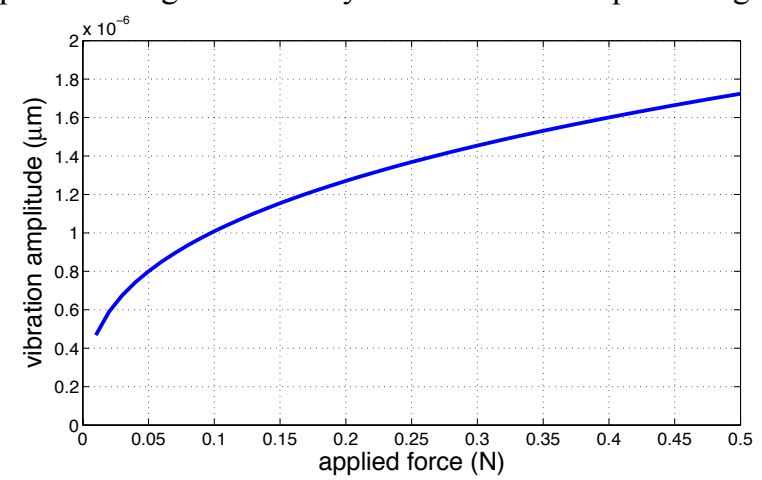

Figure 4: Detach condition of the fingerprint in function of the applied force: as an example, a $0.15 \mathrm{~N}$ applied load with a vibrational amplitude of $1.2 \mu \mathrm{m}$ is required to induce detachment.

\section{Non-Coulombic friction}

In the previous section, it was shown that detachment was possible for a range of normal vibrational amplitudes that are compatible with those commonly employed in ultrasonic devices. Assuming that there is not frictional aging, then it is possible to calculate the minimum effect on the periodic frictional force by taking account of the fact that the coefficient of friction decreases with increasing normal force for smooth surfaces [12]; this analysis is relevant for the possible application of the 
principle to the screen of smartphone and tablets. Finally, a comparison with experimental data will be made to examine the significance of the phenomenon.

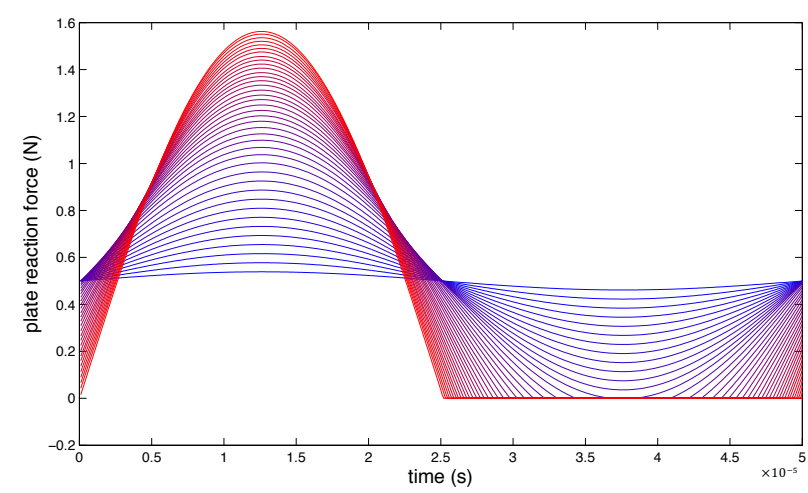

Figure 5: Plate reaction force as a function of the time over a vibration cycle for different vibrational amplitudes. The amplitude of vibration varies from $0.1 \mu \mathrm{m}$ (blue) to $8 \mu \mathrm{m}$ (red). During the detachment of the springs from the plate, the plate reaction force is zero.

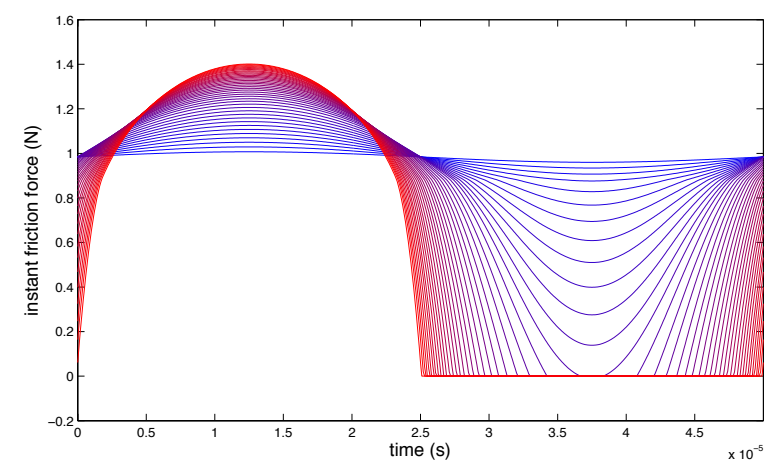

Figure 6: Instantaneous frictional force as a function of the time over a vibration cycle for different vibrational amplitudes. The amplitude of vibration varies from $0.1 \mu \mathrm{m}$ (blue) to $8 \mu \mathrm{m}$ (red). During the detachment of the springs from the plate, the frictional force is zero.

At equilibrium, the integral of the imposed force from the the bed of springs must be equal to the total reaction force of the plate over a vibration cycle:

$$
\int_{c y c l e} F_{\text {reac }} d t=F_{n} T
$$

where $F_{\text {reac }}$ is the normal reaction force of the plate in contact with the springs and $T$ is the vibrational period of the plate. The vibration of the plate is described by $\phi \sin (2 \pi f t)$, where $\phi$ is the vibrational amplitude and $f$ is the frequency. In this case, the frequency is $20 \mathrm{kHz}$ leading to a period of $50 \mu \mathrm{s}$. With this relationship it is possible to compute the instantaneous compressive force, $F_{\text {reac }}$, of the springs induced by the vibrating plate; the case shown in Fig. 5 is for an applied normal force of $0.5 \mathrm{~N}$. The corresponding frictional force, $\mu_{d} F_{\text {reac }}$, is plotted in Fig. 6 . It may be seen that the contact is frictionless for an increasing proportion of the unloading cycle as the amplitude of vibration increases.

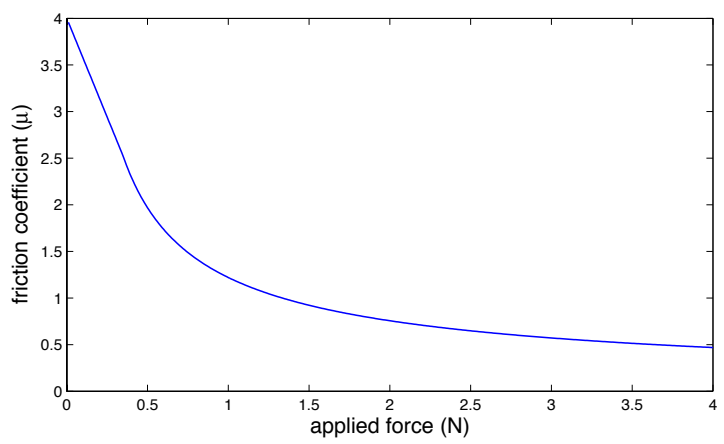

Figure 7: Coefficient of friction between a finger pad and a smooth surface as a function of the applied normal force [11]. 
The radial contact pressure profile for a finger pad in contact with a smooth flat plate is not uniform as assumed above but can be approximated by the Hertzian function:

$$
P(r)=\frac{3}{2} \frac{F_{n}}{\pi b^{2}} \sqrt{1-\frac{r^{2}}{b^{2}}}
$$

where $b$ is the radius of contact and $r$ is the radial coordinate with an origin at the center of contact. From a knowledge of $P(r)$, it is possible to integrate the reaction force across the contact over the cycle period and hence compute the mean normal force as a function of time. The frictional force can then be calculated from the following integral:

$$
F_{l}=\int_{0}^{b} \mu_{d}\left(F_{\text {reac }}\right) P(r) \pi^{2} d r
$$

where $\mu_{d}$ is a function of the normal force as given in Fig. 7. Fig. 8 shows that the calculated value of the relative coefficient of friction, $F_{l}(\phi) / F_{l}(0)$, decreases with increasing vibration amplitude.

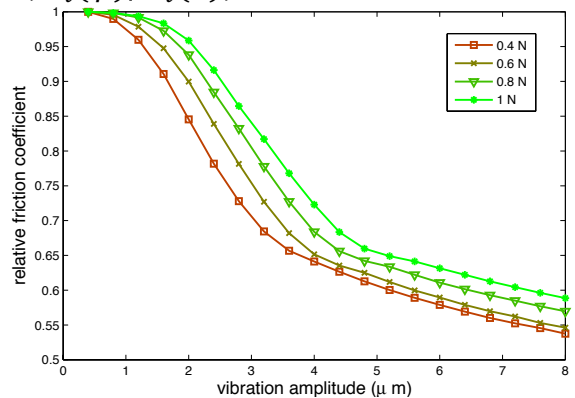

Figure 8: Relative coefficient of friction as a function of the vibrational amplitude induced by the non-linearity of the coefficient of friction.

\section{Experimental comparison}

It was possible to compare the simulated friction reduction recovered by this method with experimental data taken from [16] to highlight the compatibility of the effect with the general results observed for ultrasonic devices. In that work, the friction reduction induced by the normal ultrasonic vibrating device has been measured with a tribometer for different index fingers and for different loads applied. Fig. 9 shows a comparison of the reduction in the coefficient of friction measured for three fingers with an applied normal force of $0.35 \mathrm{~N}$ for different vibrational amplitudes. The model predicts the trend of decreasing friction with increasing amplitude as observed experimentally, even though the starting slope is mismatched. This phenomenon is due to the full elastic implementation of the simulation, the lack of a viscoelastic term leads to a poor simulation for small amplitude of vibration. It may be noticed that this approach, at the contrary of the squeeze film hypothesis, leads to a finite value of friction modulation without predicting full finger levitation.

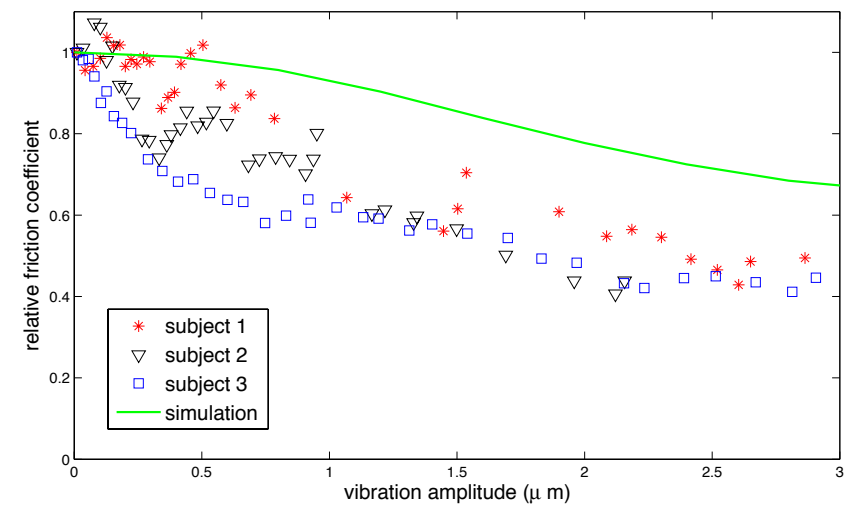

Figure 9: Experimental comparison between the modeled phenomena and experimental results taken from [16] for normal vibration; the considered frequency was $25 \mathrm{kHz}$. 


\section{DISCUSSION}

The proposed elastic model is a considerable simplification of the real system, but nevertheless can explain part of the behavior of a finger pad sliding over an ultrasonic vibrating plate. The reduction in friction has been used for the creation of different tactile stimulators [17], [18], but the underlying

mechanisms are still unresolved. The squeeze film effect has been shown to be a contributory factor [10] but the behavior of the friction modulation is not in complete agreement with the analysis proposed in [6], which suggests that there are other concomitant contributions. The currently proposed elastic model of the fingerprint ridges is consistent with a psychophysical assessment of the influence of lateral ultrasonic vibrations. In particular, it is clear that ultrasonic perturbation frequencies are essential to induce a sufficiently stiff response of the finger pad and also cause micro-slip in a temporal domain that is at least comparable to the times required to thermally reactivate a molecular junction. Published values of such times are sparse but it is interesting that, for thin molecular layers in aqueous media, repinning times are $\sim 200 \mu$ s [19], which is comparable to the period $(50 \mu \mathrm{s})$ of the vibrational frequency $(20 \mathrm{kHz})$.

In the case of normal vibrations, the results of the model are consistent with the trends observed experimentally [10]. Taking account of the non-linearity of the coefficient of friction was an important factor in the model. Again, high frequencies are a prerequisite for ensuring that the gross retardation time of the finger pad is much longer than the cycle time of the vibration. While the reduction in friction calculated with the current model was based on the separation time between a finger pad and a virtual vibrating plate, ultrasonic frequencies will induce a synergistic effect of ensuring that the disrupted molecular junction network remains at least partially disrupted even during contact. This would result in a further reduction in the coefficient as well as a possible contribution from a squeeze flow effect. It is also possible that an additional effect could be attributed to a reduction in the occlusion arising from the secreted moisture from the sweat pores (deocclusion). Essentially, the intermittent loss of contact could allow some moisture to diffuse from the contact region and hence result in asperity hardening compared with a continuous intimate contact

Simple models of the type described in the current work are useful in order to establish key principles for formulating models that are more complete. The non-linear viscoelastic behavior of the finger pad is particularly relevant since it controls the gross temporal response of the finger pad. In addition, it is important to consider the details of the contact mechanics because detachment involves radial crack propagation in which an annulus of failure grows from the periphery of a contact [13]. Consequently, different mechanisms may dominate across the contact region e.g. the squeeze flow mechanism may be localized at the periphery of the contact.

\section{CONCLUSIONS}

A simple model of the interaction of a finger pad with a plate vibrating at ultrasonic frequencies, which is based on representing the finger print ridges as elastic springs, captures some of the critical factors that govern the performance of vibrational haptic displays. In particular, it is concluded that ultrasonic frequencies are essential for ensuring that the finger pad is sufficiently stiff to cause periodic contact separation for out-of-plane vibrations. More work is required to establish the relative contributions of this mechanism, squeeze flow and deocclusion. For both normal and lateral vibrations, it is also necessary to consider the disruption of the network of molecular junctions that are responsible for friction.

\section{ACKNOWLEDGEMENTS}

The authors would like to thank Sofiane Ghenna for the useful assistance with the piezoelectric actuator. The authors would like to acknowledge FP7 Marie Curie Initial Training Network PROTOTOUCH, grant agreement No. 317100 as well as Nord Pas-de-Calais region, and FEDER within framework of Touch-it project for founding.

\section{REFERENCES}

[1] M. Wiertlewski, J. Lozada, and V. Hayward, "The Spatial Spectrum of Tangential Skin Displacement Can Encode Tactual Texture," IEEE Trans. Robot., vol. 27, no. 3, pp. 461-472, Jun. 2011.

[2] M. Biet, G. Casiez, F. Giraud, and B. Lemaire-Semail, "Discrimination of Virtual Square Gratings by Dynamic Touch on Friction Based Tactile Displays," in symposium on Haptic interfaces for virtual environment and teleoperator systems, 2008. haptics 2008, 2008, pp. 41-48.

[3] E. Vezzoli, M. Amberg, F. Giraud, and B. Lemaire-Semail, "Electrovibration Modeling Analysis," in Haptics: Neuroscience, Devices, Modeling, and Applications, M. Auvray and C. Duriez, Eds. Springer Berlin Heidelberg, 2014, pp. 369-376.

[4] O. Bau, I. Poupyrev, A. Israr, and C. Harrison, "TeslaTouch: Electrovibration for Touch Surfaces," in Proceedings of the 23Nd Annual ACM Symposium on User Interface Software and Technology, New York, NY, USA, 2010, pp. 283-292.

[5] K. A. Kaczmarek, K. Nammi, A. K. Agarwal, M. E. Tyler, S. J. Haase, and D. J. Beebe, "Polarity Effect in Electrovibration for Tactile Display," IEEE Trans. Biomed. Eng., vol. 53, no. 10, pp. 2047-2054, Oct. 2006.

[6] M. Biet, F. Giraud, and B. Lemaire-Semail, "Squeeze film effect for the design of an ultrasonic tactile plate," IEEE Trans. Ultrason. Ferroelectr. Freq. Control, vol. 54, no. 12, pp. 2678-2688, Dec. 2007.

[7] T. Watanabe and S. Fukui, "A method for controlling tactile sensation of surface roughness using ultrasonic vibration," in , 1995 IEEE International Conference on Robotics and Automation, 1995. Proceedings, 1995, vol. 1, pp. 1134-1139 vol.1.

[8] E. Vezzoli, W. Ben Messaoud, F. Giraud, and B. Lemaire-Semail, "Pressure dependence of friction modulation in ultrasonic devices," World Haptics Conference (WHC), 2015.

[9] M. Biet, F. Giraud, and B. Lemaire-Semail, "Implementation of tactile feedback by modifying the perceived friction," Eur. Phys. J. - Appl. Phys., vol. 43, no. 01, pp. 123-135, Jul. 2008. 
[10] X. Dai, J. E. Colgate, and M. A. Peshkin, "LateralPaD: A surface-haptic device that produces lateral forces on a bare finger," in 2012 IEEE Haptics Symposium (HAPTICS), 2012, pp. 7-14.

[11] A. N. Gent, G. R. Hamed, and W. J. Hung, "Adhesion of elastomers: Dwell time effects," J. Adhes., vol. 79, no. 4, pp. 315-325, Apr. 2003.

[12] B. M. Dzidek, M. Adams, Z. Zhang, S. Johnson, S. Bochereau, and V. Hayward, "Role of Occlusion in Non-Coulombic Slip of the Finger Pad," in Haptics: Neuroscience, Devices, Modeling, and Applications, M. Auvray and C. Duriez, Eds. Springer Berlin Heidelberg, 2014, pp. $109-116$.

[13] M. Wiertlewski and V. Hayward, "Mechanical behavior of the fingertip in the range of frequencies and displacements relevant to touch," $J$. Biomech., vol. 45, no. 11, pp. 1869-1874, Jul. 2012.

[14] V. A. Ho and S. Hirai, "A novel model for assessing sliding mechanics and tactile sensation of human-like fingertips during slip action," Adv. Tactile Sens. Touch-Based Hum. Robot Interact., vol. 63, Part 3, no. 0, pp. 253-267, Jan. 2015.

[15] M. J. Adams, S. A. Johnson, P. Lefèvre, V. Lévesque, V. Hayward, T. André, and J.-L. Thonnard, "Finger pad friction and its role in grip and touch," J. R. Soc. Interface, vol. 10, no. 80, p. 20120467, Mar. 2013.

[16] T. Sednaoui, E. Vezzoli, B. M. Dzidek, B. Lemaire-Semail, C. Chiappaz, and M. Adams, "Experimental evaluation of friction reduction in ultrasonic devices," in World Haptics Conference (WHC), 2015.

[17] M. Amberg, F. Giraud, B. Semail, P. Olivo, G. Casiez, and N. Roussel, "STIMTAC: A Tactile Input Device with Programmable Friction," in Proceedings of the 24th Annual ACM Symposium Adjunct on User Interface Software and Technology, New York, NY, USA, 2011, pp. 7-8.

[18] L. Winfield, J. Glassmire, J. E. Colgate, and M. Peshkin, "T-PaD: Tactile Pattern Display through Variable Friction Reduction," in EuroHaptics Conference, 2007 and Symposium on Haptic Interfaces for Virtual Environment and Teleoperator Systems. World Haptics 2007. Second Joint, 2007, pp. 421426.

[19] C. Drummond, J. Israelachvili, and P. Richetti, "Friction between two weakly adhering boundary lubricated surfaces in water," Phys. Rev. E, vol. 67, no. 6, p. 066110, Jun. 2003. 